\title{
Integrated Optical Fibre Coupler
}

\author{
Christopher Holmes, Alexander Jantzen, Stephen G Lynch, Paul C Gow, Teresa Ferreiro, \\ Peter GR Smith
}

University of Southampton, Southampton, S017 1BJ, UK

Integrated Optical Fibre (IOF) is a low-loss photonic platform that directly integrates optical fibre to a planar substrate. The platform possess advantages associated with optical fibre including low propagation loss, whilst at the same time enabling planar functionality associated with integrated optics. IOF fabrication is achieved through a modified Flame Hydrolysis Deposition (FHD) technique that forms a robust glass alloy between the fibre and substrate. The binding medium is of optical quality and mechanically robust, illustrated in fig 1 (a). Although non-trivial seamless on/off-chip coupling has also been demonstrated, which could further act to minimise coupling losses typically associated with integrated optics.

Thus far, developments in IOF have largely utilised either environmental stability [1] and/or physical robustness [2] and not realised the formats potential for optical integration. This work reports for the first time an integrated optical fibre coupler, illustrated in figure 1(b)-(e). The proof-of-concept device could be scaled and serve as a building block for future large-scale low-loss integrated architectures. For example it could be feasible to concatenate multiple coupler components with integrated functionality e.g. a thermo-optic tuning capability.
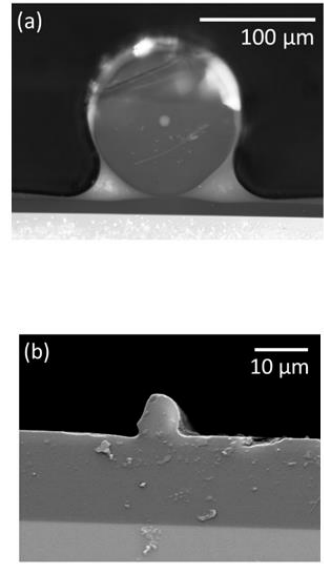
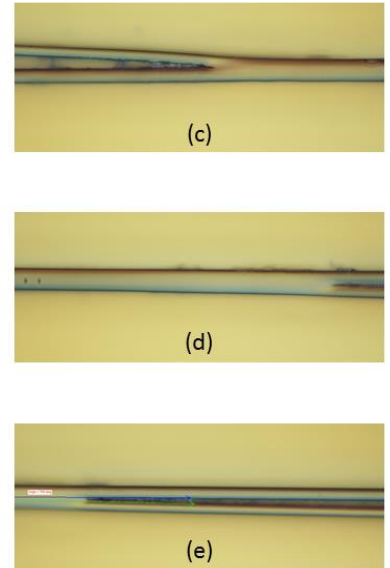
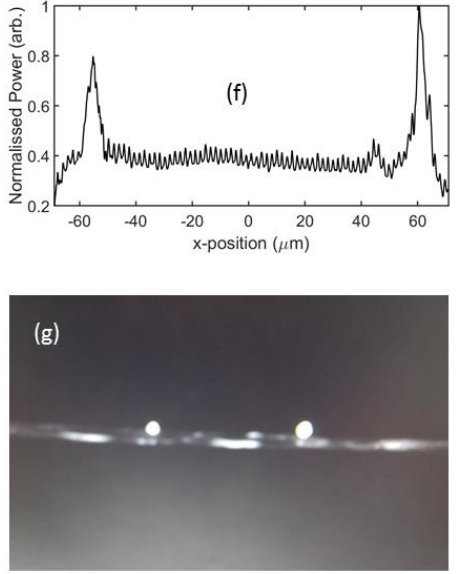

Fig. 1 (a) the cross-section of integrated optical fibre, with SMF-28 (b) a similar cross-section with SMF-28 pre-etched to an $8 \mu \mathrm{m}$ diameter, enabling evanescent field exposure (c-e) top view optical microscope image of coupler, showing $8 \mu \mathrm{m}$ diameter fibres entering a coupling region. (f) Intensity plot attained from a CCD camera image of the end facet after a single arm launch of $780 \mathrm{~nm}$ wavelength and (g) IR camera image upon single arm input coupling at $780 \mathrm{~nm}$ wavelength.

To achieve this proof-of-concept component it was necessary to expose the evanescent field of the optical fibre, such that two fibres could optically couple. Exposure was achieved through use of a wet etching, using a buffered HF solution. In the etching of this structure the diameter of SMF-28 fibre was brought down to $8 \mu \mathrm{m}$. After etching a pair of such fibres were layered-up upon a silicon wafer. The silicon wafer had a thick thermally grown oxide $(15 \mu \mathrm{m})$ that acted as an optical buffer (clad), ensuring a guided mode did not leak into the silicon substrate. The layer-up stage brought the two etched fibres into physical contact and thus enabled directional coupling. In this initial demonstration a $0.5 \mu \mathrm{m}$ thick FHD layer was deposited and consolidated about the fibre(s), illustrated in Fig 1(b)-(e). The FHD silicate was doped with boron and phosphorus such that the refractive index matched that of the thermal oxide and it had a consolidation temperature below $1250^{\circ} \mathrm{C}$. It is noted that due to meniscus effects during consolidation the thickness of FHD varies about the respective fibres. The component demonstrated brought two fibres together at an angle of 3-degrees, after which they are in physical proximity for $\sim 1 \mathrm{~mm}$ and then depart at an angle similar to input, illustrated in Figure 1 (c)-(e). Upon single arm input illumination at $780 \mathrm{~nm}$ wavelength a 34:66 splitting ratio was attained, illustrated in Figure 1 (f)-(g). Improvements to surface roughness of the etched fibres, optimised FHD thickness and refractive index and fibre diameter insertion losses should be further reduced.

This work will report the latest results for propagation losses in etched fibre after IOF layer-up and the insertion losses brought about for optimised coupler designs. Comment shall be made on routes for scalability of this technique.

\section{References}

[1] S. G. Lynch, C. Holmes, S. A. Berry, C. James, A. Jantzen, T. I. Ferreiro, and P. G. R. Smith, "External cavity diode laser based upon an FBG in an integrated optical fiber platform," Opt. Ex., 24, 8, pp. 22781-22788, (2016).

[2] C. Holmes, J. C. Gates, and P. G. R. Smith, "Planarised optical fiber composite using flame hydrolysis deposition demonstrating an integrated FBG anemometer," Opt. Express, 22, 26, p. 32150, (2014). 